\title{
Cultural adaptation of Internet- and mobile- based interventions for mental disorders: a systematic review protocol
}

\author{
Kerstin Spanhel ${ }^{1^{*}} \mathbb{D}$, Sumeyye Balci ${ }^{2}$, Harald Baumeister ${ }^{2}$, Juergen Bengel ${ }^{1}$ and Lasse B. Sander ${ }^{1}$
}

\begin{abstract}
Background: Internet- and mobile-based interventions (IMI) are an effective and scalable low-threshold solution to reach people who are undersupplied by current healthcare. Adapting interventions to the cultural and ethnic background of the target group enhances their acceptance and effectiveness. However, no systematic approach to cultural adaptation of IMI has been established so far. Therefore, this review aims to summarise components and procedures commonly used in the cultural adaptation of IMI for mental disorders, as well as the current evidence base on whether such a cultural adaptation leads to an increased acceptance, adherence, and effectiveness of IMI for mental disorders.
\end{abstract}

Methods: A systematic literature search will be performed using the following databases: MEDLINE, Embase, PsycINFO, CENTRAL, and WoS. The search term will include keywords related to cultural adaptation, IMI, and mental disorders/disturbances. Two independent reviewers will evaluate studies against inclusion and exclusion criteria and extract study and intervention characteristics, details on the cultural adaptation approach, and outcome data. Quality of evidence will be assessed using the Quality Assessment Tool for Reviewing Studies with Diverse Designs, and results will be synthesised qualitatively.

Discussion: Providing adequate mental healthcare regardless of cultural backgrounds is a major global health challenge. The planned systematic review will lay the foundation for the further development of the cultural adaptation of IMI for mental disorders by summarising the current state and providing recommendations for future research.

Systematic review registration: PROSPERO CRD42019142320

Keywords: Mental health gap, Cultural sensitive psychotherapy, eHealth, Low-threshold intervention

\footnotetext{
* Correspondence: kerstin.spanhel@psychologie.uni-freiburg.de

'Department of Rehabilitation Psychology and Psychotherapy, Institute of

Psychology, University of Freiburg, Engelbergerstr. 41, 79085 Freiburg,

Germany

Full list of author information is available at the end of the article
}

(C) The Author(s). 2020 Open Access This article is licensed under a Creative Commons Attribution 4.0 International License, which permits use, sharing, adaptation, distribution and reproduction in any medium or format, as long as you give appropriate credit to the original author(s) and the source, provide a link to the Creative Commons licence, and indicate if changes were made. The images or other third party material in this article are included in the article's Creative Commons licence, unless indicated otherwise in a credit line to the material. If material is not included in the article's Creative Commons licence and your intended use is not permitted by statutory regulation or exceeds the permitted use, you will need to obtain permission directly from the copyright holder. To view a copy of this licence, visit http://creativecommons.org/licenses/by/4.0/ The Creative Commons Public Domain Dedication waiver (http://creativecommons.org/publicdomain/zero/1.0/) applies to the data made available in this article, unless otherwise stated in a credit line to the data. 


\section{Background}

Health inequalities remain a major global health challenge [1-3]. Particularly, migrants living in high-income countries and the general population of low- and middle-income countries (LMIC) are affected by a reduced availability of health services. This holds particularly true for mental health: although migrants often face various stressors before, during, and after migration [4], it has been found that they are less likely to use mental health services and, if they use them, find them less helpful $[5,6]$. Similarly, a tremendous treatment gap in mental healthcare in LMIC has been revealed, i.e. the prevalence of mental disorders highly outnumbers the use of health services. It is estimated that, in LMIC, about $76-85 \%$ of people with a serious mental disorder do not receive any treatment, whereas about $35-50 \%$ receive no treatment in high-income countries [7-9]. Reasons for these mental health inequalities are linked to structural as well as individual barriers. Structural barriers include a small number of available mental health services and a poor accessibility of existing services [1012]. Individual barriers include language and cultural barriers (e.g. understanding of disease and treatment processes, stigmatisation of mental disorders), a lack of information about the healthcare system, and a negative attitude towards the healthcare system [10-13].

The World Health Organization has called to take action in order to adequately reach these specific populations that migrated from or live in LMIC and to hereby achieve an improved global health equality [9]. This goal can only be reached when both individual and structural barriers are addressed. To reduce structural barriers, Internet- and mobile-based interventions (IMI) have been suggested as a low-threshold solution that enhances the accessibility of health services for a broad range of people in need [13-20]. The advantages of IMI include their anonymity, temporal and local independence, easy accessibility, and scalability [21-25]. The effectiveness of IMI has been proven in prevention, treatment, and aftercare for various mental disorders [25-29]. However, IMI are mostly developed and evaluated for people living in high-income countries, and they seem to be less effective for people with a differing ethnic background [30]. Thus, individual barriers to IMI have to be addressed simultaneously. Cultural adaptation of psychological interventions [31-34], i.e. the consideration of "language, culture, and context in such a way that it is compatible with the client's cultural patterns, meanings, and values" ( [35]; p. 362), has been suggested to reduce existing individual barriers $[36,37]$ and to enhance their acceptance, relevance, and effectiveness [3841]. Thus, culturally adapting IMI may be key to increase their reach and impact in culturally diverse people $[19,42]$. In fact, in recent years, various research groups have culturally adapted IMI for people with diverse cultural backgrounds [43-47], which has been found to enhance the effectiveness of the IMI in the target groups [48]. Most research groups hereby relied on frameworks for the cultural adaptation of face-to-face treatments [31, 49-51], which, however, may not be valid when adapting IMI [52]. Previous reviews and meta-analyses have summarised studies that conducted IMI in LMIC [53] and investigated the extent and effectiveness of cultural adaptation of IMI for the treatment of common mental disorders among people with diverse cultural backgrounds [48]. Yet, the procedure of the cultural adaptation is often only poorly reported [43, 52, 54-56]. This makes it difficult to identify components of the cultural adaptation that may contribute to an enhanced acceptance and effectiveness of IMI, such as the language, illustrations, or example characters $[43,54,56,57]$.

Hence, in this review, we will systematically identify and summarise methods previously used for the cultural adaptation of IMI for mental disorders. We will answer the following research questions:

1) Which components are commonly considered in the cultural adaptation of IMI for mental disorders?

2) Which are procedures that are commonly used in the cultural adaptation of IMI for mental disorders?

Furthermore, we will summarise the current evidence base on whether cultural adaptation of IMI for mental disorders leads to an increased acceptance, adherence, and effectiveness of such interventions.

Thus, this review will inform future researchers and developers working on the cultural adaptation of IMI for a mental disorder. The cultural adaptation of IMI holds great potential to reduce both individual and structural barriers to treatment-seeking, which might contribute to reduce global mental health inequalities.

\section{Methods/design}

This protocol has been developed in line with the Preferred Reporting Items for Systematic Review and MetaAnalysis Protocol (PRISMA-P) statement [58] (see Additional file 1). The systematic review has been registered with the International Prospective Register of Systematic Reviews (PROSPERO) database (https://www.crd.york.ac. uk/PROSPERO; registration number: CRD42019142320), and the findings will be reported using the Preferred Reporting Items for Systematic Review and Meta-Analysis (PRISMA) guidelines [59].

\section{Eligibility criteria}

Studies will be chosen in line with the criteria outlined below and in Table 1. 
Table 1 Study inclusion criteria

\begin{tabular}{|c|c|}
\hline Participants & $\begin{array}{l}\text { (a) People with a cultural (i.e. national or ethnic) } \\
\text { background differing from the initial target group } \\
\text { of the intervention }\end{array}$ \\
\hline Intervention & $\begin{array}{l}\text { (b) Adapted to the target group } \\
\text { (c) Psychological methods to address mental } \\
\text { disorders/disturbances } \\
\text { (d) In an Internet-, computer-, or mobile-based } \\
\text { setting }\end{array}$ \\
\hline Comparator & (e) With/without a control group \\
\hline Outcomes & (f) Illustration of the cultural adaptation approach \\
\hline Study type & $\begin{array}{l}\text { (g) All study types (e.g. with/without follow-up, } \\
\text { qualitative/quantitative) }\end{array}$ \\
\hline Setting & (h) No restrictions in the type of setting \\
\hline Report characteristics & $\begin{array}{l}\text { (i) No restriction in the publication year } \\
\text { (j) No restriction in language } \\
\text { (k) Peer-reviewed journal articles }\end{array}$ \\
\hline
\end{tabular}

Studies are eligible for inclusion (a) if the target group differs from the target group of the original intervention in terms of culture, i.e. nationality or ethnicity. (b) Interventions must have been adapted to the target group. If the intervention was only translated but not otherwise culturally adapted, it will be excluded. (c) Interventions must address mental health issues and must be based on psychological interventions. The term psychological intervention is defined according to Kampling et al. ( [60], p.2): "Psychological interventions aim to recognise, improve or prevent distress by direct or interactive communication." They include cognitive behaviour therapy, psychodynamic psychotherapy, behaviour therapy or behaviour modification, systemic therapy, third-wave cognitive behavioural therapies, humanistic therapies, integrative therapies, and other psychological-oriented interventions. General health promotion interventions will not be included. (d) Interventions must predominantly be provided in an Internet-, computer-, or mobilebased setting. (e) Studies both with and without a control group will be included in the review. (f) Studies will be included if an illustration of the methods to culturally adapt the intervention is provided in the manuscript or-upon request-by the authors. (g) All study types, i.e. controlled and non-controlled trials, qualitative and quantitative studies, studies with or without a follow-up assessment, will be included. (h) There will be no restrictions by type of setting. (i) Neither will there be a restriction in the publication year, $(j)$ nor in the language. Articles will be translated to English. (k) Only peer-reviewed journal articles will be included to guarantee a high quality of the extracted data. We assume that the information in grey literature will not significantly affect our research questions, as sufficient information will be available in peer-reviewed literature. In order to save resources, grey literature will thus be excluded.

\section{Search strategy}

Electronic databases will be searched to identify published work, including MEDLINE, PsycINFO, Cochrane Central Register of Controlled Trials (CENTRAL), Embase, and Web of Science (WoS). A comprehensive search strategy developed by the project team will be used, which will include medical subject headings (MeSH terms) and text words related to the key elements of cultural adaptation of interventions in an Internet-, computer-, or mobile-based setting. The search term will include keywords of a parallel systematic review for the cultural adaptation of IMI in the context of health promotion. The search will not be limited. We used a validation set of 13 studies to test the sensitivity of the search term. The test search yielded a coverage of $100 \%$. The full search term for PsycINFO can be found in Additional file 2. To complement the search, backward searches of the reference lists as well as forward searches of the identified relevant publications will be conducted.

\section{Study records Data management}

Literature search results will be uploaded to covidence, a software for reference management that also facilitates the communication among the reviewers. It will be used to identify and remove duplicates, to categorise publications according to their inclusion or exclusion, as well as for data extraction and quality assessment.

\section{Study selection process}

The selection of articles will be conducted by two independent reviewers (KS, SB). In a first step, all titles and abstracts yielded by the search will be screened in duplicate against the inclusion criteria. Full texts of the articles of all titles and abstracts that seem to meet the inclusion criteria or of which there is any uncertainty will be obtained. In a second step, these full texts will be screened in duplicate in terms of the eligibility criteria. Disagreement on including articles will be resolved in discussion; if further needed, a third reviewer (LS) will be consulted. Thus, selected articles will be searched backward and forward; new articles will be screened in the alike way. Reasons for exclusion will be recorded. Neither of the reviewers will be blind to the journal titles or to the study authors or institutions.

A PRISMA flow chart [59] will illustrate the study selection process (see Fig. 1).

\section{Data extraction process}

The following information will be extracted from each article by an independent reviewer (KS) using an extraction form, if the information is available: (a) study identification items (first author, year of publication, type of 


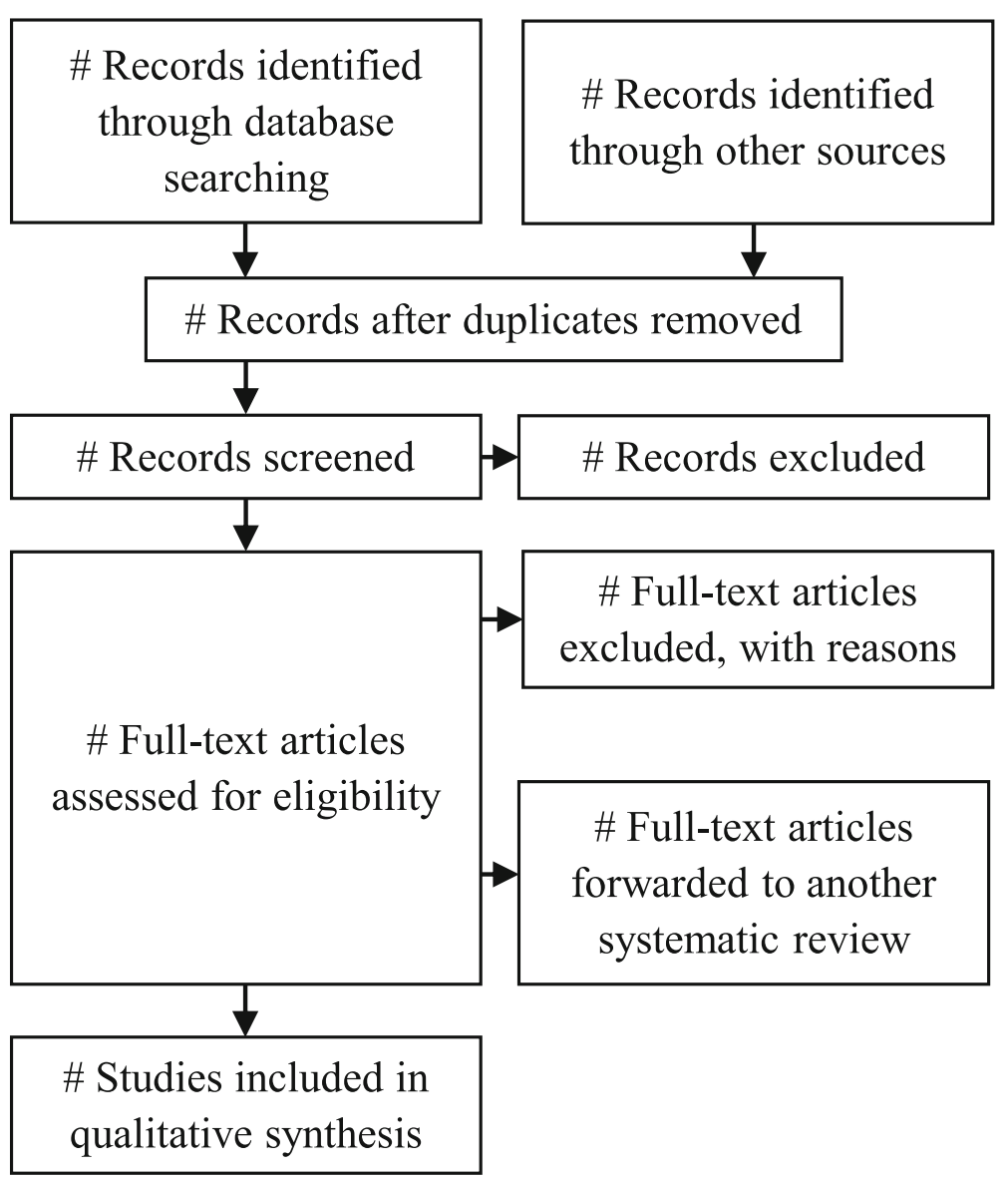

Fig. 1 Study selection process (adapted from [59])

document), (b) sample characteristics (sample/group size, age, gender, country of origin, ethnicity), (c) study design characteristics (control group: no/non-adapted/ delayed intervention or treatment as usual, randomisation, type of assessments, follow-up assessments), (d) study settings (country of study conduction, recruitment strategy), (e) original intervention characteristics (language, type, target group: gender/age/cultural background, target mental disorder, delivery mode, level of human support/guidance, duration of intervention and modules, components of intervention, signposting to other interventions), (f) culturally adapted intervention characteristics (language, type, target group: gender/age/ cultural background, target mental disorder, delivery mode, level of human support/guidance, duration of intervention and modules, components of intervention, signposting to other interventions), (g) details on cultural adaptation approach (language, persons, metaphors, content, concepts, goals, methods, context, design, structure, functionality, aesthetics, open aspects), details on the process of cultural adaptation (feasibility/ pilot trial, literature review, qualitative methods, persons involved), and illustration of an orientation towards a guideline of cultural adaptation of face-to-face treatments (name of authors, name of guidelines), as well as (h) outcomes (participation and attrition rates: maximum/minimum/median number of sessions completed, primary outcomes/secondary outcomes: which outcomes were used, tools used to measure outcomes, baseline symptom severity for all outcomes, findings/outcomes as measured by authors). We will contact the study authors to resolve any uncertainty and receive missing information, if the variables of interest are not available or not clearly presented in the studies. All extracted data will be double-checked by a second independent reviewer (SB).

\section{Quality assessment}

Two independent reviewers (KS, SB) will assess the Quality Assessment Tool for Reviewing Studies with Diverse Designs (QATSDD) [61]. The quality of the following criteria will be evaluated: (1) explicit theoretical framework, (2) statements of aims/objectives in main body of report, (3) clear description of research setting, (4) evidence of sample size considered in terms of analysis, (5) representative sample of target group of a reasonable size, (6) description of procedure for data 
collection, (7) rationale for choice of data collection tool(s), (8) detailed recruitment data, (9) statistical assessment of reliability and validity of measurement tool(s) (quantitative only), (10) fit between stated research question and method of data collection (quantitative), (11) fit between stated research question and format and content of data collection tool (qualitative), (12) fit between research question and method of analysis, (13) good justification for analytical method selected, (14) assessment of reliability of analytical process (qualitative), (15) evidence of user involvement in design, and (16) strengths and limitations critically discussed.

The quality of the studies will be rated against the criteria on a four-point scale, producing a global score showing a low or high quality. If discrepancies arise between the two reviewers, they will be resolved in discussion; if further needed, a third reviewer (LS) will be consulted.

\section{Data synthesis}

A narrative synthesis of all included articles will be conducted. Thereby, the results will be described in detail in text and tables, including the characteristics and findings of the studies (i.e. extracted data). Similarities and links between the included studies will be explored. The narrative synthesis will inform the first and second research questions by providing a qualitative report on the procedure of cultural adaptation and hereby considered components. Findings related to the effectiveness of, acceptance of, and adherence to the culturally adapted IMI for mental disorders will be displayed, if possible, in comparison to the non-adapted IMI.

\section{Meta-biases}

If study protocols are available, we will compare the outcomes reported in the protocol and in the published report in order to examine the potential for selective outcome reporting. If not available, outcomes reported in the methods and result sections of the published reports will be compared.

\section{Discussion}

Previous research suggests that culturally adapting psychological interventions [40, 41] as well as IMI [48] may enhance their effectiveness in populations differing from the initial target group. Whereas there are a number of guidelines for the cultural adaptation of face-to-face treatments, no consistent procedure exists in culturally adapting IMI. This systematic review will provide a summary of the cultural adaptation of IMI for mental disorders, which can enable an improved methodological procedure. Specific components and procedures that are suggested to be important to adapt will be identified by comparing previously conducted studies. Additionally, the acceptance and effectiveness of culturally adapted IMI for mental disorders will be investigated, and, if possible, compared to the non-adapted IMI. However, the heterogeneity in clinical, methodological, and statistical approaches may limit the interpretability. This review will include trials that may differ on the addressed mental disorders, cultural backgrounds of the target population, methods, interventions, control groups, assessments, and outcomes. Due to being a rather young research field and the linked assumption of a limited amount of relevant studies, we aim to conduct a broad review including heterogeneous studies. By contrast, studies in which culturally sensitive IMI for mental disorders were developed without the adaptation of an earlier version will not be included in the review; this is due to the assumption that the development of such an intervention requires other aspects than its adaptation.

The planned synthesis of the current state of cultural adaptation of IMI for mental disorders will lay the foundation for its further development, informing both researcher and developer of IMI. This will help in making IMI impactful for people living in or migrating from LMIC and contribute to delivering behavioural health services worldwide.

\section{Supplementary information}

Supplementary information accompanies this paper at https://doi.org/1 0.1186/s13643-020-01438-y

Additional file 1. PRISMA-P 2015 checklist.

Additional file 2. PsycINFO search term.

Abbreviations

CENTRAL: Cochrane Central Register of Controlled Trials; IMI: Internet- and mobile-based interventions; LMIC: Low- and middle-income countries; MeSH: Medical subject headings; PRISMA: Preferred Reporting Items for Systematic Review and Meta-Analysis; PRISMA-P: Preferred Reporting Items for Systematic Review and Meta-Analysis Protocol; PROSPERO: Prospective Register of Systematic Reviews; QATSDD: Quality Assessment Tool for Reviewing Studies with Diverse Designs; WoS: Web of Science

\section{Acknowledgements}

We would like to thank the Studienstiftung des deutschen Volkes for financing the first author's PhD scholarship.

Authors' contributions

$\mathrm{KS}, \mathrm{SB}, \mathrm{HB}, \mathrm{JB}$, and LBS conceived the study design. KS is the guarantor of the review. KS, SB, and LBS developed the search strategy. HB and LBS provided methodological expertise. KS wrote the draft of the manuscript. All authors read, provided feedback on, and approved the final manuscript.

\section{Funding}

The article processing charge was funded by the German Research Foundation (DFG) and the Albert Ludwigs University Freiburg in the funding programme Open Access Publishing. Other than that, this research did not receive any specific grant from funding agencies in the public, commercial, or not-for-profit sectors.

Availability of data and materials Not applicable. 


\section{Ethics approval and consent to participate}

Not applicable.

\section{Consent for publication}

Not applicable.

\section{Competing interests}

$\mathrm{KS}, \mathrm{SB}$, and JB declare that they have no competing interests. $\mathrm{HB}$ received consultancy fees, reimbursement of congress attendance, and travel costs as well as payments for lectures from Psychotherapy and Psychiatry Associations as well as Psychotherapy Training Institutes in the context of E-Mental-Health topics. He has been the beneficiary of study support (third-party funding) from several public funding organizations. LBS received personal fees from Psychotherapy Training Institutes and clinics in the context of E-Mental-Health topics and supervision outside the submitted work.

\section{Author details}

${ }^{1}$ Department of Rehabilitation Psychology and Psychotherapy, Institute of Psychology, University of Freiburg, Engelbergerstr. 41, 79085 Freiburg, Germany. ${ }^{2}$ Department of Clinical Psychology and Psychotherapy, Institute of Psychology and Education, Ulm University, Lise-Meitner-Str. 16, 89081 UIm, Germany.

Received: 20 November 2019 Accepted: 29 July 2020

\section{Published online: 03 September 2020}

\section{References}

1. Braveman P. Health disparities and health equity: concepts and measurement. Annu Rev Public Health. 2005;27(1):167-94.

2. Fiscella K, Franks P, Gold MR, Clancy CM. Inequality in quality: addressing socioeconomic, racial, and ethnic disparities in health care. JAMA. 2003; 283(19):2579-84.

3. Goesling B, Firebaugh G. The trend in international health inequality. Popul Dev Rev. 2004;30(1):131-46.

4. Spallek J, Zeeb H, Razum O. What do we have to know from migrants' past exposures to understand their health status? A life course approach. Emerg Themes Epidemiol. 2011;8(1):6.

5. Brzoska P, Razum O. Erreichbarkeit und Ergebnisqualität rehabilitativer Versorgung bei Menschen mit Migrationshintergrund [Accessibility and quality of rehabilitative services among migrants in Germany]. Bundesgesundheitsblatt - Gesundheitsforsch - Gesundheitsschutz. 2015; 58(6):553-9.

6. Razum O, Zeeb H, Meesmann U, Schenk L, Bredehorst M, Brzoska P, et al. Schwerpunktbericht der Gesundheitsberichterstattung des Bundes: Migration und Gesundheit [Federal focus report on health: Migration and health]. Berlin: Robert-Koch-Institut; 2008. p. 138.

7. World Health Organization. Prevalence, severity, and unmet need for treatment of mental disorders in the World Health Organization world mental health surveys. J Am Med Assoc. 2004;291 (21):2581-90.

8. Patel V. Mental health in low- and middle-income countries. Br Med Bull. 2007:81-82(1):81-96.

9. World Health Organization. Mental health action plan 2013-2020. 2013.

10. Eaton J, McCay L, Semrau M, Chatterjee S, Baingana F, Araya R, et al. Scale up of services for mental health in low-income and middle-income countries. Lancet. 2011;378(9802):1592-603.

11. Patel V, Chowdhary N, Rahman A, Verdeli H. Improving access to psychological treatments: lessons from developing countries. Behav Res Ther. 2011:49(9):523-8.

12. Saraceno B, van Ommeren M, Batniji R, Cohen A, Gureje O, Mahoney J, et al. Barriers to improvement of mental health services in low-income and middle-income countries. Lancet. 2007;370(9593):1164-74.

13. Bockting CLH, Williams AD, Carswell K, Grech AE. The potential of low intensity and online interventions for depression in low- and middleincome countries. Glob Ment Heal. 2016;3:e25.

14. IASC Inter-Agency Standing Committee. IASC guidelines on mental health and psychosocial support in emergency settings. Geneva: IASC; 2007. p. 1-103.

15. Hinton DE, Jalal B. Guidelines for the implementation of culturally sensitive cognitive behavioural therapy among refugees and in global contexts. Intervention. 2014;12:78-93.
16. Sijbrandij M. Expanding the evidence: key priorities for research on mental health interventions for refugees in high-income countries. Epidemiol Psychiatr Sci. 2017;27(2):105-8.

17. Schneider F, Bajbouj M, Heinz A. Psychische Versorgung von Flüchtlingen in Deutschland: Modell für ein gestuftes Vorgehen [Mental treatment of refugees in Germany. Model for a stepped approach]. Nervenarzt. 2017; 88(1):10-7.

18. Wagner B. Online-Therapie - eine neue Perspektive in der Psychotherapie für Flüchtlinge und Asylbewerber? [Online-therapy - a new perspective in psychotherapy with refugees and asylumseekers?]. Psychother Forum. 2016; 21(4):124-31.

19. Muñoz RF, Bunge EL, Chen K, Schueller SM, Bravin Jl, Shaughnessy EA, et al. Massive open online interventions: a novel model for delivering behavioralhealth services worldwide. Clin Psychol Sci. 2016;4(2):194-205.

20. Mohr DC, Schueller SM, Araya R, Gureje O, Montague E. Mental health technologies and the needs of cultural groups. The Lancet Psychiatry. 2014; 1(5):326-7.

21. Griffiths F, Lindenmeyer A, Powell J, Lowe P, Thorogood M. Why are health care interventions delivered over the Internet? A systematic review of the published literature. J Med Internet Res. 2006;8(2):e10.

22. Carroll KM, Rounsaville BJ. Computer-assisted therapy in psychiatry: be brave - it's a new world. Curr Psychiatry Rep. 2010;12(5):426-32.

23. Moock J. Support from the Internet for individuals with mental disorders: advantages and disadvantages of e-mental health service delivery. Front Public Heal. 2014;2(1):65-70.

24. Andersson G, Titov N. Advantages and limitations of Internet-based interventions for common mental disorders. World Psychiatry. 2014;13(1):4-11.

25. Ebert DD, Van Daele T, Nordgreen T, Karekla M, Compare A, Zarbo C, et al. Internet and mobile-based psychological interventions: applications, efficacy and potential for improving mental health. Eur Psychol. 2018;23(2):167-87.

26. Carlbring P, Andersson G, Cuijpers P, Riper H, Hedman-Lagerlöf E. Internetbased vs. face-to-face cognitive behavior therapy for psychiatric and somatic disorders: an updated systematic review and meta-analysis. Cogn Behav Ther. 2018:47(1):1-18.

27. Hedman E, Ljótsson B, Lindefors N. Cognitive behavior therapy via the Internet: a systematic review of applications, clinical efficacy and costeffectiveness. Expert Rev Pharmacoecon Outcomes Res. 2012;12(6):745-64.

28. Sander $L$, Rausch $L$, Baumeister $H$. Effectiveness of internet-based interventions for the prevention of mental disorders: a systematic review and meta-analysis. JMIR Ment Heal. 2016;3(3):e38.

29. Hennemann S, Farnsteiner S, Sander L. Internet- and mobile-based aftercare and relapse prevention in mental disorders: a systematic review and recommendations for future research. Internet Interv. 2018;14:1-17.

30. Karyotaki E, Ebert DD, Donkin L, Riper H, Twisk J, Burger S, et al. Do guided internet-based interventions result in clinically relevant changes for patients with depression? An individual participant data meta-analysis. Clin Psychol Rev. 2018;63:80-92.

31. Joyce C, Leino A. Advancement in the maturing science of cultural adaptations of evidence-based interventions. J Consult Clin Psychol. 2017;85(1):45-57.

32. Bernal G, Saéz-Santiago E. Culturally centered psychosocial interventions. J Community Psychol. 2006;34(2):121-32.

33. Sue S, Zane N. The role of culture and cultural techniques in psychotherapy: a critique and reformulation. Am Psycho. 1987:42(1):37-45.

34. Rogler LH. The meaning of culturally sensitive research in mental health. Am J Psychiatry. 1989;146(3):296-303.

35. Bernal G, Jiménez-Chafey MI, Domenech Rodríguez MM. Cultural adaptation of treatments: a resource for considering culture in evidence-based practice. Prof Psychol Res Pract. 2009;40(4):361-8.

36. Gearing RE, Schwalbe CS, Mackenzie MJ, Brewer KB, Ibrahim RW, Olimat HS, et al. Adaptation and translation of mental health interventions in Middle Eastern Arab countries: a systematic review of barriers to and strategies for effective treatment implementation. Int J Soc Psychiatry. 2013;59(7):671-81.

37. Sue S, Zane N, Nagayama Hall GC, Berger LK. The case for cultural competency in psychotherapeutic interventions. Annu Rev Psychol. 2009; 60(1):525-48.

38. Barrera M, Castro FG, Strycker LA, Toobert DJ. Cultural adaptations of behavioral health interventions: a progress report. J Consult Clin Psychol. 2013;81(2):196-205.

39. Benish SG, Quintana S, Wampold BE. Culturally adapted psychotherapy and the legitimacy of myth: a direct-comparison meta-analysis. J Couns Psychol. 2011;58(3):279-89. 
40. Chowdhary N, Jotheeswaran AT, Nadkarni A, Hollon SD, King M, Jordans MJD, et al. The methods and outcomes of cultural adaptations of psychological treatments for depressive disorders: a systematic review. Psychol Med. 2014:44(6):1131-46.

41. Griner D, Smith TB. Culturally adapted mental health interventions: a metaanalytic review. Psychother Theory, Res Pract. 2006:43(4):531-48.

42. Heim E, Harper Shehadeh M, van't Hof E, Carswell K. Cultural adaptation of scalable interventions. In: Maercker A, Heim E, Kirmayer $\sqcup$, editors. Cultural clinical psychology and PTSD. Boston: Hogrefe Publishing; 2019. p. 201-18.

43. Juniar D, van Ballegooijen W, Karyotaki E, van Schaik A, Passchier J, Heber E, et al. Web-based stress management program for university students in Indonesia: systematic cultural adaptation and protocol for a feasibility study. JMIR Res Protoc. 2019;8(1):e11493.

44. Choi I, Zou J, Titov N, Dear BF, Li S, Johnston L, et al. Culturally attuned internet treatment for depression amongst Chinese Australians: a randomised controlled trial. J Affect Disord. 2012;136(3):459-68.

45. Kayrouz R, Dear BF, Karin E, Fogliati VJ, Titov N. A pilot study of a clinicianguided internet-delivered cognitive behavioural therapy for anxiety and depression among Arabs in Australia, presented in both English and Arabic languages. Int Interv. 2016;5:5-11.

46. Knaevelsrud C, Brand J, Lange A, Ruwaard J, Wagner B. Web-based psychotherapy for posttraumatic stress disorder in war-traumatized Arab patients: randomized controlled trial. J Med Internet Res. 2015; 17(3):e71.

47. Wang Z, Wang J, Maercker A. Chinese My Trauma Recovery, a web-based intervention for traumatized persons in two parallel samples: randomized controlled trial. J Med Internet Res. 2013;15(9):112-25.

48. Harper Shehadeh M, Heim E, Chowdhary N, Maercker A, Albanese E. Cultural adaptation of minimally guided interventions for common mental disorders: a systematic review and meta-analysis. JMIR Ment Heal. 2016;3(3): e44.

49. Bernal G, Bonilla J, Bellido C. Ecological validity and cultural sensitivity for outcome research - issues for the cultural-adaptation and development of psychosocial treatments with Hispanics. J Abnorm Child Psychol. 1995;23(1): 67-82.

50. Helms JE. An examination of the evidence in culturally adapted evidencebased or empirically supported interventions. Transcult Psychiatry. 2015; 52(2):174-97.

51. Hwang WC. The formative method for adapting psychotherapy (FMAP): a community-based developmental approach to culturally adapting therapy Prof Psychol Res Pract. 2009;40(4):369-77.

52. Lal S, Gleeson J, Malla A, Rivard L, Joober R, Chandrasena R, et al. Cultural and contextual adaptation of an ehealth intervention for youth receiving services for first-episode psychosis: adaptation framework and protocol for horyzons-Canada phase 1. JMIR Res Protoc. 2018;20(4):e100.

53. Arjadi R, Nauta MH, Chowdhary N, Bockting CLH. A systematic review of online interventions for mental health in low and middle income countries: a neglected field. Glob Ment Heal. 2015;2:e12.

54. Abi Ramia J, Harper Shehadeh M, Kheir W, Zoghbi E, Watts S, Heim E, et al. Community cognitive interviewing to inform local adaptations of an emental health intervention in Lebanon. Glob Ment Heal. 2018;5:e39.

55. Arjadi R, Nauta MH, Suryani AO, Bockting CLH. Guided Act and Feel Indonesia - Internet-based behavioral activation intervention for depression in Indonesia: a systematic cultural adaptation. Makara Hubs Asia. 2018;22(1):3-11.

56. Salamanca-Sanabria A, Richards D, Timulak L. Adapting an internet-delivered intervention for depression for a Colombian college student population: an illustration of an integrative empirical approach. Internet Interv. 2019;15:76-86.

57. Spanhel K, Schweizer JS, Wirsching D, Lehr D, Baumeister H, Bengel J, et al. Cultural adaptation of internet interventions for refugees: Results from a user experience study in Germany. Internet Interv. 2019;18:100252.

58. Shamseer L, Moher D, Clarke M, Ghersi D, Liberati A, Petticrew M, et al. Preferred reporting items for systematic review and meta-analysis protocols (PRISMA-P) 2015: elaboration and explanation. BMJ. 2015;349:g7647.

59. Liberati A, Altman DG, Tetzlaff J, Mulrow C, Gøtzsche PC, loannidis JPA, et al. The PRISMA statement for reporting systematic reviews and meta-analyses of studies that evaluate health care interventions: explanation and elaboration. J Clin Epidemiol. 2009;62(10):e1000100.
60. Kampling $\mathrm{H}$, Baumeister $\mathrm{H}$, Jackel WH, Mittag O. Prevention of depression in chronically physically ill adults. Cochrane Database Syst Rev. 2014;2014(8): CD011246.

61. Sirriyeh $\mathrm{R}$, Lawton $\mathrm{R}$, Gardner $\mathrm{P}$, Armitage $\mathrm{G}$. Reviewing studies with diverse designs: the development and evaluation of a new tool. J Eval Clin Pract. 2012;18(4):746-52

\section{Publisher's Note}

Springer Nature remains neutral with regard to jurisdictional claims in published maps and institutional affiliations.
Ready to submit your research? Choose BMC and benefit from:

- fast, convenient online submission

- thorough peer review by experienced researchers in your field

- rapid publication on acceptance

- support for research data, including large and complex data types

- gold Open Access which fosters wider collaboration and increased citations

- maximum visibility for your research: over $100 \mathrm{M}$ website views per year

At BMC, research is always in progress.

Learn more biomedcentral.com/submissions 